\title{
Diseño de una prueba de ejecución de estrategias para el aprendizaje de estudiantes universitarios
}

\section{Design of a test execution of strategies for learning of university students}

\author{
Daniel González Lomelí \\ María de los Ángeles Maytorena Noriega \\ César Walterio Varela Romero \\ Rosario Leticia Domínguez Guedea \\ Universidad de Sonora
}

\begin{abstract}
Resumen
El objetivo del presente estudio fue diseñar y validar una prueba sobre estrategias de aprendizaje en un contexto de ejecución a partir de un modelo multidimensional de evaluación. A través de un Análisis Cognoscitivo de Tarea (ACT), se determinaron los tipos de contenidos a ser evaluados y las operaciones cognoscitivas que subyacen tras el aprendizaje del contenido del texto Estrategias para aprender a aprender de la materia del mismo nombre, de una universidad pública del noroeste de México. A partir de los resultados del ACT, se elaboró una prueba de Estrategias Para el Aprendizaje (EPA) constituida con 28 reactivos, la cual se aplicó a una muestra intencional de 144 estudiantes de licenciatura. Un análisis Rasch dio como resultado una prueba de 26 reactivos, posteriormente, se realizaron análisis factoriales confirmatorios, por medio de modelamiento por ecuaciones estructurales, por tipos de conocimientos (declarativo y procedimental) y por contexto de ejecución (reconocimiento y recuerdo); se obtuvieron dos modelos con ajuste adecuado a los datos de acuerdo con los índices más aceptados. Los estudiantes de ingeniería obtuvieron un mayor desempeño en la prueba EPA al ser evaluados en el contexto de recuerdo, en comparación con los de psicología.

Palabras clave: Estrategias de aprendizaje, prueba de ejecución, diseño y validación, estudiantes universitarios, evaluación.
\end{abstract}

Nota del autor

Daniel González Lomelí, Posgrado Integral en Ciencias Sociales, Universidad de Sonora; María de los Ángeles Maytorena Noriega, Departamento de Psicología, Universidad de Sonora; César Walterio Varela, Departamento de Psicología, Universidad de Sonora; Rosario Leticia Domínguez Guedea, Departamento de Psicología, Universidad de Sonora.

Esta investigación fue subvencionada por el Consejo Nacional de Ciencia y Tecnología de México a través de la convocatoria de Ciencia Básica 2011/167235.

La correspondencia en relación con este artículo debe dirigirse a Daniel González Lomelí, Posgrado Integral en Ciencias Sociales, Universidad de Sonora, Hermosillo, Sonora, 83000.

Dirección electrónica:dgonzalez@investigacion.uson.mx 


\begin{abstract}
The objective of this study was to design and validate a test on learning strategies, in an execution context from a multidimensional model of evaluation. Through an Analysis of Cognitive Task (ACT) identified the types of content to be evaluated and the cognitive operations that underlie learning the content of the text Strategies to learn how to learn on the subject of the same name, a public university in the northwest of Mexico. From the results of the ACT was developed a test of Learning Strategies (EPA) formed with 28 reagents, which are applied to an intentional sample of 144 undergraduate students. A Rasch analysis resulted in a test of 26 reagents, subsequently were confirmatory factorial analysis, through structural equation modeling, by types of knowledge (declarative and procedural) and by execution context (recognition and remembrance); two models were obtained with appropriate adjustment to the data according to the indices more accepted. Engineering students gain a greater performance in the EPA test to be evaluated in the context of remembrance, in comparison with those of psychology.
\end{abstract}

Keywords: Learning strategies, test execution, design and validate, university students, evaluation.

Desde finales del siglo veinte investigaciones ligadas a la problemática estudiantil en México destacan la existencia de altos índices de demanda escolar (Martínez-Guerrero \& Sánchez-Sosa, 1993), reprobación, solicitudes de cambio de carrera, deserción escolar, bajas cifras de titulación (González, Valenzuela, \& Maytorena, 1999) y una apreciable desorientación vocacional (Aguilar, Pacheco, Andrade, Vargas, Gutiérrez, \& Zetina, 1992) de los jóvenes, máxime durante los primeros semestres de la licenciatura.

En su estudio, López-Olivas (2007), concluye que:

por los resultados observados en los exámenes de admisión, la imagen de alumno que éstos reflejan realmente, puede percibirse como alejada de la que cabría considerar deseable para un centro educativo, teniendo presentes las debilidades que pueden advertirse en las pruebas empleadas para realizar dicho proceso. La indefinición de las variables que se intentan examinar también es preocupante, ya que si el objeto de la medición no se hace explícito y si su vaguedad no orienta acerca de las técnicas óptimas para proceder a su medición, el proceso en su conjunto estará debilitado (p. 33).

Los esfuerzos de las instituciones educativas por solucionar estos problemas no se han hecho esperar (Universidad de Sonora, 2004), por ejemplo, a partir de los lineamientos generales para un modelo curricular de la Universidad de Sonora (UNISON), se define en el espacio curricular Estrategias para Aprender a Aprender (EAA), correspondiente al eje de formación común, cuyo propósito es el desarrollo de habilidades intelectuales para el estudio mediante el aprendizaje de estrategias cognitivas para la búsqueda, adquisición, organización, análisis 
y uso de la información y del conocimiento, el desarrollo de las habilidades de expresión oral y escrita, el desarrollo de la creatividad y el pensamiento crítico. Así, la asignatura EAA tiene como objetivo general (Universidad de Sonora, 2004):

que el estudiante desarrolle estrategias cognoscitivas y metacognoscitivas que le permitan adaptarse a exigencias del trabajo académico universitario, con base en el análisis crítico de los materiales de estudio, cuyainformación puede abordarse en diferentes niveles de abstracción. El dominio de dichas estrategias se llevará a cabo a través de sus conocimientos, comprensión y constantes aplicaciones.

Aquí surge una pregunta, ¿Cómo se podrán identificar los aprendizajes asociados a la materia EEA?

De ahí que sea una necesidad prioritaria que los procesos de evaluación que deseen dar cuenta de los resultados de los aprendizajes de los estudiantes, sean sometidos a una serie de análisis rigurosos de su contenido o dominio, validez y su capacidad de predicción (Castañeda, 2006). En este contexto, resulta especialmente pertinente contar con estrategias estadísticas confirmatorias que permitan verificar las presunciones fundamentales de las pruebas de medición.

En el ámbito internacional, Castañeda (2006) reconoce que la calidad educativa y la evaluación de resultados de aprendizaje son interdependientes y están íntimamente ligadas: los puntajes de los exámenes muestran la urgente necesidad de efectuar reformas sustanciales en la política y la práctica educativas en vías de mejorar la ejecución de profesores y estudiantes, así como la efectividad de planes y programas de estudio. Se considera necesario tener siempre presente que la validez de las evaluaciones está limitada por nuestra comprensión, tanto del desempeño académico como de la estructuración de los dominios específicos a evaluar.

Dos de los conceptos implicados en el aprendizaje estratégico son los referidos a las estrategias de aprendizaje y la autorregulación. Las estrategias de aprendizaje son actividades deliberadas que dirigen las acciones a realizar para el logro de las metas de aprendizaje establecidas. Valle, González, Cuevas y Rodríguez (2003) señalan que las estrategias de aprendizaje engloban los recursos cognoscitivos que emplea el estudiante cuando aprende; así mismo incluye las actividades de planificación, dirección, control (autorregulación), además de aspectos motivacionales.

Mientras que, según De Jong y Simons (1990) el aprendizaje autorregulado: consiste en el uso de las estrategias y actividades de aprendizaje adecuadas a las metas planteadas. Un aprendiz autorregulado es capaz de cambiar con flexibilidad entre diferentes actividades de aprendizaje, dependiendo de las metasy restricciones delatarea. Escapaz de ejecutar actividades de aprendizaje 
que lo lleven al conocimiento, comprensión, integración y a la solución de problemas (p. 83).

Dentro de los modelos explicativos del aprendizaje estratégico se encuentra el Modelo de Aprendizaje Estratégico de Castañeda (1998), el cual posee:

1) Una porción de fomento que comprende las estrategias cognoscitivas de aprendizaje (adquisición, recuperación y procesamiento de la información) y las estrategias de autorregulación (de persona, de la tarea por aprender y de los materiales de estudio) y;

2) Una porción de evaluación que tiene un Modelo Multidimensional de Evaluación (MME) para el aprendizaje, conformado por las dimensiones de contenidos a ser evaluados (declarativo, procedimental, condicional), los contextos de recuperación (reconocimiento y recuerdo) y los procesos cognoscitivos subyacentes al aprendizaje.

En este modelo la autora afirma que las estrategias son mediadores del aprendizaje y son afectadas por los procesos de autorregulación. En el Modelo Multidimensional de Evaluación de Castañeda, los dos contextos de recuperación de información tienen como objetivo identificar los efectos diferenciales de las demandas asociadas con la variedad de niveles de complejidad subyacente a la ejecución de diversos contextos de prueba. Usar de manera eficiente distintos contextos de prueba permite construir herramientas evaluativas sensibles a la detección de fallas y apoya la prescripción oportuna de técnicas remediales específicas e individualizadas (Castañeda, 1998, 2004; González, Castañeda, \& Maytorena, 2009). Asimismo, el evaluador selecciona el nivel de dificultad en el que se debe evaluar el contenido de acuerdo con el objetivo de aprendizaje y diseña aquellas preguntas que lo evalúen cuidando que la prueba sea válida y sensible a las posibles fallas de los estudiantes en los diferentes niveles de complejidad del contenido.

La complejidad de los procesos cognoscitivos subyacentes a la ejecución evalúa diversos niveles de demanda: desde los más simples (discriminación), hasta los asociados a la generalización, la categorización conceptual, la integración del conocimiento y la solución de problemas. Cada una de estas dimensiones pone a prueba los diferentes niveles de dificultad del contenido, de recuperación de la información y de los procesos subyacentes, por lo cual el evaluador deberá tener muy claro qué y cómo medir para un determinado objetivo instruccional. Se requiere elaborar exámenes que integren las interacciones necesarias entre las tres dimensiones. Así, será capaz de determinar qué, cómo y en qué tipo de tarea un estudiante dado falló y dar la instrucción remedial necesaria o rediseñar las condiciones del aprendizaje.

A partir de lo anterior, suponemos que sí se construye un aprueba de ejecución sobre estrategias de aprendizaje a partir del MME para el aprendizaje, entonces se podrá diferenciar y 
retroalimentar el rendimiento de los estudiantes en cuanto a tipos de conocimiento y contextos de recuperación de información.

Con el objetivo de medir las estrategias de aprendizaje que aplican los estudiantes universitarios, se diseñó y elaboró una prueba de ejecución basada en el MME, a partir del contenido del libro Estrategias para Aprender a Aprender (EAA), utilizado como texto en el espacio curricular del mismo nombre de una universidad pública del noroeste de México. La finalidad era tener una medida confiable, válida y socialmente pertinente de las poblaciones de interés en este estudio.

\section{Análisis Cognoscitivo de Tarea (ACT)}

Como afirma Castañeda (2004), el ACT: permite analizar la actividad a ser evaluada en pasos específicos para identificar, en una secuencia progresiva de mayor nivel de detalle y precisión, los conocimientos, habilidades y valores asociados a cada paso. Requiere establecer descripciones claras de los conocimientos semánticos (conceptual), procedimentales (cómo llevar a cabo acciones) y estratégicos (establecer metas, seleccionar procedimientos y controlar avances), mediante una descomposición recursiva de las tareas involucradas $\mathrm{y}$ tomando en cuenta los niveles diferenciales de complejidad incluidos en cada tipo de conocimiento (p. 68).
Como resultado del ACT realizado a partir del contenido del libro mencionado, se logró identificar para cada una de las 4 unidades que lo integran, los tipos de conocimientos a ser evaluados y las operaciones cognoscitivas asociadas a dichos conocimientos (tabla 1). La Unidad I: Autonomía para el aprendizaje sólo posee conocimientos de tipo conceptual y las operaciones cognoscitivas de identificar y clasificar. La Unidad II: Proceso de comprensión de lectura contiene conocimientos de tipo conceptual, procedimental y condicional, relacionados con procesos cognoscitivos que van desde identificar, clasificar, dar orden temporal, hasta traducir y aplicar procedimientos. La Unidad III: Estrategias de aprendizaje para la construcción de textos y el desarrollo del pensamiento crítico posee conocimientos de tipo conceptual y operaciones cognoscitivas de identificar y de aplicación de procedimientos. La Unidad IV: Aplicación de estrategias cognoscitivas y metacognoscitivas en la producción de ensayos contiene conocimientos conceptuales y procedimentales y procesos cognoscitivos de identificación y de aplicación de procedimientos.

Posteriormente, se generaron los indicadores y se elaboraron los reactivos. La prueba contiene 28 reactivos (Anexo) con cuatro opciones de respuesta y sólo una respuesta correcta, la cual se codificó como uno y las respuestas incorrectas con cero; para las unidades I, II, III y IV se elaboraron 7, 7, 5 y 9 reactivos, respectivamente, que se distribuyen en dos niveles de dificultad 
Tabla 1

Análisis cognitivo de tarea del texto Estrategias para aprender a aprender: Conocimientos a ser evaluados y operaciones cognoscitivas identificadas

\begin{tabular}{lll}
\hline Unidad & Conocimiento & Proceso cognoscitivo \\
\hline $\begin{array}{ll}\text { I. Autonomía para el } \\
\text { aprendizaje }\end{array}$ & Conceptual & $\begin{array}{l}\text { Identificación } \\
\text { Clasificación }\end{array}$ \\
$\begin{array}{ll}\text { II. Procesos de } \\
\text { comprensión de } \\
\text { textos }\end{array}$ & $\begin{array}{l}\text { Conceptual } \\
\text { Identificación } \\
\text { Clasificación }\end{array}$ \\
$\begin{array}{ll}\text { Condimental } \\
\text { aprendizaje para la } \\
\text { construcción de } \\
\text { textos y el desarrollo } \\
\text { del pensamiento } \\
\text { crítico }\end{array}$ & Conceptual & $\begin{array}{l}\text { Ordenar temporalmente } \\
\text { Tracción }\end{array}$ \\
$\begin{array}{l}\text { IV. Aplicación de } \\
\text { estrategias } \\
\text { cognoscitivas y } \\
\text { metacognoscitivas } \\
\text { en la producción de } \\
\text { ensayos }\end{array}$ & & $\begin{array}{l}\text { Aplicación de procedimientos } \\
\text { Identificación } \\
\text { Aplicación de procedimientos }\end{array}$ \\
\hline
\end{tabular}

Tabla 2

Distribución de reactivos de la prueba por tipo de conocimiento y contexto de recuperación

\begin{tabular}{|c|c|c|c|}
\hline \multirow{2}{*}{$\begin{array}{l}\text { Tipo de } \\
\text { conocimiento }\end{array}$} & \multirow{2}{*}{$\begin{array}{l}\text { Proceso } \\
\text { cognoscitivo }\end{array}$} & \multicolumn{2}{|c|}{ Contexto } \\
\hline & & Reconocimiento & Recuerdo \\
\hline \multirow[t]{2}{*}{ Declarativo } & Identificar & $\begin{array}{l}\text { R2, R3, R7, R8, R9, } \\
\text { R11, R20, R24, R25, R27, } \\
\text { R26, }\end{array}$ & \\
\hline & Clasificar & $\begin{array}{l}\text { R1, R4, R5, R6, R10, } \\
\text { R21, R23, }\end{array}$ & $\begin{array}{l}\text { R17, R18, } \\
\text { R22, }\end{array}$ \\
\hline Procedimental & $\begin{array}{l}\text { Traducir } \\
\text { Inferir } \\
\text { Aplicar }\end{array}$ & & $\begin{array}{l}\text { R13, R16, R19, } \\
\text { R15, } \\
\text { R12, R14, R28 }\end{array}$ \\
\hline
\end{tabular}


en la recuperación de lo aprendido (tabla 2); 18 reactivos en el nivel fácil, asociado al contexto de reconocimiento, donde la misma evaluación permite que el estudiante recupere lo aprendido; y 10 reactivos en el nivel difícil o contexto de recuerdo, el cual plantea al estudiante un nivel extra de procesamiento para la construcción de la respuesta. Es el mismo estudiante quien debe construirlos a partir de procesos cognoscitivos con el fin de tener éxito en la recuperación necesaria de la información aprendida (Castañeda, 1999; González et al., 2009).

\section{Método}

\section{Participantes}

A través de un muestreo no aleatorio intencional se seleccionó una muestra de 144 estudiantes de una licenciatura del área de Ciencias Sociales $(n=69)$ y de una licenciatura del área de Ingeniería $(n=75)$ de tercer semestre.

\section{Instrumento}

La prueba de estrategias para el aprendizaje construida a partir de los contenidos del libro de texto Estrategias para Aprender a Aprender (Araoz, Guerrero, Galindo, Villaseñor, \& De la Vara, 2010), contiene 28 reactivos; 18 en el contexto de Recuperación o fácil y 10 reactivos en el contexto de Recuerdo o difícil (Anexo).
- Ejemplos de reactivos por operación cognoscitiva:

Comprender y organizar información: R20. Texto mediante el cual se expone, analiza, reflexiona, argumenta y comenta una interpretación personal sobre un tema determinado:
a) Ensayo
b) Hipertexto
c) Apéndice
d) Resumen

Aplicar conceptos principios y procedimientos: R12. Elabora un mapa conceptual a partir del siguiente texto:

- Ejemplo de reactivos por contexto de recuperación de la información:

c) Reconocimiento (nivel fácil):

R27. Marca la opción que enlista en orden los pasos para realizar un hipertexto:

a) Lectura en grupos del material e identificación de ideas principales, revisar individualmente y comentar lo leído, repetir la lectura y las discusiones y decidir el nombre de la problemática elegida.

b) Lectura en grupos del material e identificación de ideas principales, revisar individualmente y comentar lo leído y decidir el nombre de la problemática elegida.

c) Lectura en grupos del material, revisar individualmente y comentar lo leído, repetir la lectura y las discusiones y decidir el nombre de la problemática elegida.

d) Lectura en grupos del material, decidir el nombre de la problemática elegida, elaborar un mapa conceptual y escribir claramente. 
Recuerdo (nivel difícil):

R19. "El amor de la madre significa dicha, paz, no hace falta conseguirlo ni merecerlo. La relación con el padre es enteramente distinta". ¿Por qué el argumento expuesto es una falacia?:

\section{Procedimiento}

La aplicación de la prueba, elaborada ex profeso para este estudio, se realizó en los salones de clases en los horarios de cada grupo que consintió participar de manera voluntaria e informada después de haber explicado los objetivos del proyecto, leída y firmada la carta de Consentimiento Informado.

\section{Análisis de datos}

Con el propósito de determinar las propiedades métricas y la unidimensionalidad de la escala Estrategias para el aprendizaje, se llevó a cabo el análisis de los 28 reactivos de respuesta dicotómica que la componen, aplicando los criterios de interpretación recomendados (Wright \& Stone, 1979) de ajuste interno y externo, el valor de discriminación y el punto biserial, utilizando el software winsteps v.3.7 (Linacre, 2006). Asimismo, para conocer la información que producen los reactivos se siguieron los estimadores sugeridos por la literatura especializada (Cadavid, Delgado, \& Prieto, 2007; González, 2008; Prieto \& Delgado, 2003).

Se realizó un primer Análisis Factorial Confirmatorio (AFC) con el fin de construir un modelo bifactorial de estrategias para el aprendizaje por tipos de conocimientos (Castañeda, 1998, 2004; González et al., 2009) a través del Modelamiento por Ecuaciones Estructurales (MEE, Bentler, 2006). Posteriormente, se realizó otro AFC a través del MEE (Bentler, 2006) con el fin de construir un modelo bifactorial de estrategias para el aprendizaje en dos contextos de recuperación de la información (Castañeda, 1998, 2004; González et al., 2009).

\section{Resultados}

El resultado del ACT permitió el diseño de una prueba de ejecución que mide EPA, construida a partir de los contenidos del libro de texto Estrategias para aprender a aprender (Araoz et al., 2010), el cual contiene 28 reactivos; 18 en el contexto de Recuperación o fácil y 10 reactivos en el contexto de Recuerdo o difícil. Posteriormente, se realizó un análisis de dimensionalidad de la escala EPA a partir de análisis Rasch y los AFC a través del modelamiento por ecuaciones estructurales, por tipo de conocimientos y por contexto de recuperación de la información.

\section{Estructura general de la escala}

La medida de dificultad de la escala oscila entre el 2.49 y - 2.72 lógitos con un valor de media de 0.00 y una desviación estándar de 0.21 . Los valores promedio de ajuste interno $(1.00 s d=0.19)$ y ajuste externo $(1.01 s d=0.32)$ 
sugieren que los reactivos se encuentran dentro de los rangos aceptables de producción de información, en lo general. El valor de fiabilidad con la prueba Kuder-Richardson 20 (KR - 20) es de .97 , lo que sugiere una alta consistencia entre los reactivos que componen la escala. La amplitud del rango indica una distribución equilibrada en términos de nivel de dificultad.

\section{Análisis de estimadores de dificultad-} habilidad, ajuste y discriminación por reactivo

Considerando el valor absoluto de los criterios de ajuste, $92.8 \%$ de los reactivos muestran un ajuste interno y externo aceptable, se puede interpretar que los reactivos han sido suficientemente sensibles a los casos de determinismo o sesgo en las respuestas por obviedad (infit), así como muestran ser sensibles a valores extremos en los datos (outfit).

En la base de 28 reactivos en escalamiento se aplicó el procedimiento a 26 , ya que dos de ellos (reactivo 18 y reactivo 26) presentaron variabilidad nula, por consiguiente el procedimiento automatizado los identifica y los excluye.

Se ha considerado el valor de la discriminación empírica del reactivo, mismo que se espera tenga valores muy cercanos a 1 ; esto es descrito como el parámetro que ilustra la fuerza con que un ítem distingue a las personas con niveles del rasgo $(\theta)$ por debajo o por encima del umbral (Linacre, 1991). Los 26 reactivos medidos presentan valores aceptables.
En la tabla 3 se muestran, también, los valores de correlación de reactivo con la medida, esto implica una forma de constatar la consistencia de los reactivos en relación con la escala y los reactivos. Propiamente se advierte que los coeficientes son muy bajos; $28.5 \%$ de los reactivos muestra valores de correlación negativos, lo que sugiere en todos los sentidos la necesidad de revisar la redacción del reactivo como primera acción correctiva.

Un parámetro que se ha incluido en el presente análisis es el de la asíntota inferior (LOWER), utilizado por lo general para datos de origen de respuesta múltiple (Hambleton \& Swaminathan, 1985; Revuelta \& Ponsoda, 1998), incluso aplica para este caso que señala los reactivos susceptibles de responderse correctamente al azar. En este caso, sólo el reactivo 7 presenta un valor de asíntota (.515) inferior cercano a 1 , lo cual sugiere revisar el contenido y redacción del reactivo (el criterio marca $\geq 1$ ).

En la tabla 4 se muestran los valores de varianza cruda explicada por la medida dificultad-habilidad: global de la escala, por las habilidades de las personas que respondieron y por la dificultad-localización del reactivo. En los tres casos, el porcentaje de varianza empírica es alejada de la modelada. Igualmente, se muestra la porción de la varianza no explicada que sobrepasa la explicada.

\section{Análisis factorial confirmatorio de la prueba estrategias para el aprendizaje}

Un primer AFC permitió construir un modelo bifactorial de estrategias para el aprendizaje 
Tabla 3

Criterios de dimensionalidad y estructura de los reactivos

\begin{tabular}{|c|c|c|c|c|c|c|c|c|c|}
\hline $\begin{array}{c}\text { Medida } \\
\text { de } \\
\text { dificultad }\end{array}$ & $\begin{array}{l}\text { Núm. de } \\
\text { sujetos }\end{array}$ & $\begin{array}{c}\text { Núm. sujetos } \\
\text { respondieron } \\
1\end{array}$ & Infit & Outfit & PBSE & $\begin{array}{c}\text { DISCRI } \\
M\end{array}$ & Adivinación & Descuido & $\begin{array}{c}\text { Nombre } \\
\text { del } \\
\text { reactivo }\end{array}$ \\
\hline 2.49 & 144 & 8 & 1.036 & 1.2134 & -.097 & .9639 & .0051 & 1 & A17 \\
\hline 1.34 & 144 & 22 & 1.0534 & 1.1904 & -.1061 & .924 & .0176 & 1 & A19 \\
\hline 1.24 & 144 & 24 & 1.0124 & 1.0103 & .0154 & .9873 & .003 & 1 & A2 \\
\hline 1.09 & 144 & 27 & .957 & .894 & .1461 & 1.0639 & 0 & 1 & A28 \\
\hline 1.04 & 144 & 28 & 1.0284 & 1.0295 & -.0124 & .9667 & .0083 & 1 & A16 \\
\hline .91 & 144 & 31 & 1.053 & 1.0992 & -.0766 & .9116 & .0223 & 1 & A11 \\
\hline .86 & 144 & 32 & 1.0092 & 1.0088 & .0262 & .9871 & .0033 & 1 & A24 \\
\hline .82 & 144 & 33 & 1.0615 & 1.1779 & -.1093 & .8676 & .0336 & .2292 & A8 \\
\hline .82 & 144 & 33 & 1.0168 & 1.0291 & .0047 & .969 & .008 & 1 & A15 \\
\hline .63 & 144 & 38 & .9946 & 1.0526 & .049 & .9942 & .0006 & .3042 & $\mathrm{~A} 3$ \\
\hline .63 & 144 & 38 & 1.08 & 1.1435 & -.1247 & .8211 & .0449 & .2639 & A27 \\
\hline .31 & 144 & 47 & 1.0554 & 1.0549 & -.0615 & .8343 & .0382 & .752 & A25 \\
\hline .21 & 144 & 50 & 1.0023 & .9882 & .0445 & 1.0039 & 0 & 1 & A22 \\
\hline .05 & 144 & 55 & .9925 & .9843 & .0614 & 1.0449 & 0 & .6845 & $\mathrm{~A} 10$ \\
\hline .05 & 144 & 55 & .9623 & .9582 & .1231 & 1.1765 & 0 & 1 & A12 \\
\hline-.04 & 144 & 58 & .9763 & .9523 & .0957 & 1.1632 & 0 & .9052 & A5 \\
\hline-.19 & 144 & 63 & .9613 & .95 & .1282 & 1.2814 & 0 & 1 & A9 \\
\hline-.28 & 144 & 66 & .9659 & .9583 & .1158 & 1.2715 & 0 & 1 & A4 \\
\hline-.46 & 144 & 72 & .9325 & .9218 & .1862 & 1.5841 & 0 & 1 & A1 \\
\hline-.7 & 144 & 80 & .9777 & .9699 & .0955 & 1.1724 & .0038 & 1 & A14 \\
\hline-1.01 & 144 & 90 & .9433 & .9352 & .1505 & 1.2579 & 0 & 1 & A23 \\
\hline-1.13 & 144 & 94 & 1.0307 & 1.0427 & -.0154 & .8801 & .5159 & .984 & A7 \\
\hline-1.3 & 144 & 99 & .9787 & .9645 & .0849 & 1.0669 & 0 & 1 & A21 \\
\hline-2.21 & 144 & 121 & .9547 & .8337 & .1693 & 1.0693 & 0 & 1 & $\mathrm{~A} 13$ \\
\hline-2.44 & 144 & 125 & .9864 & .9296 & .0664 & 1.0172 & 0 & 1 & A6 \\
\hline-2.72 & 144 & 129 & 1.0053 & .9154 & .0534 & 1.0068 & 0 & 1 & A20 \\
\hline
\end{tabular}

Tabla 4

Varianza residual estandarizada en unidades de autovalor

\begin{tabular}{|c|c|c|c|}
\hline & & Empírica & Modelado \\
\hline Total de la varianza explicada en observaciones & $=35.3$ & $100.0 \%$ & $100.0 \%$ \\
\hline Varianza explicada por la medida de dificultad & $=9.3$ & $26.3 \%$ & $26.1 \%$ \\
\hline Varianza explicada por los respondientes & $=1.9$ & $5.3 \%$ & $5.3 \%$ \\
\hline Varianza explicada por los ítems & $=7.4$ & $21.0 \%$ & $20.8 \%$ \\
\hline Varianza no explicada (total) & $=26.0$ & $73.7 \%$ & $73.9 \%$ \\
\hline
\end{tabular}


por tipos de conocimientos (figura 1), el factor Conocimiento declarativo aglutina seis reactivos con pesos factoriales que van de .187 a .409, mientras que el factor Conocimiento procedimental contiene cuatro reactivos con pesos factoriales de.178 a .858, tal relación indica validez de constructo convergente para ambos contextos. La covarianza entre ambos factores o variables latentes fue significativa con un valor igual a .146 , pero menor a los pesos factoriales de cada variable manifiesta y sus respectivas variables latentes, como teóricamente era esperado, lo cual denota validez de constructo divergente entre ambas medidas. La $\chi^{2}$ fue igual a 15 (31 g.l.) con una probabilidad asociada igual a .99 , los indicadores de bondad de ajuste prácticos también fueron adecuados a los datos de acuerdo con los índices más aceptados (Bentler, 2006). El coeficiente de contingencia RHO fue igual a .516 .

El resultado del segundo AFC permitió construir un modelo bifactorial de estrategias para el aprendizaje en dos contextos de recuperación de la información (figura 2), el factor Contexto de reconocimiento aglutina cuatro reactivos con pesos factoriales que van de .202 a .510 , mientras que el factor Contexto de recuerdo contiene cuatro reactivos con pesos factoriales de .355 a .739 , esto significa validez de constructo convergente para ambos contextos. La covarianza entre ambos factores

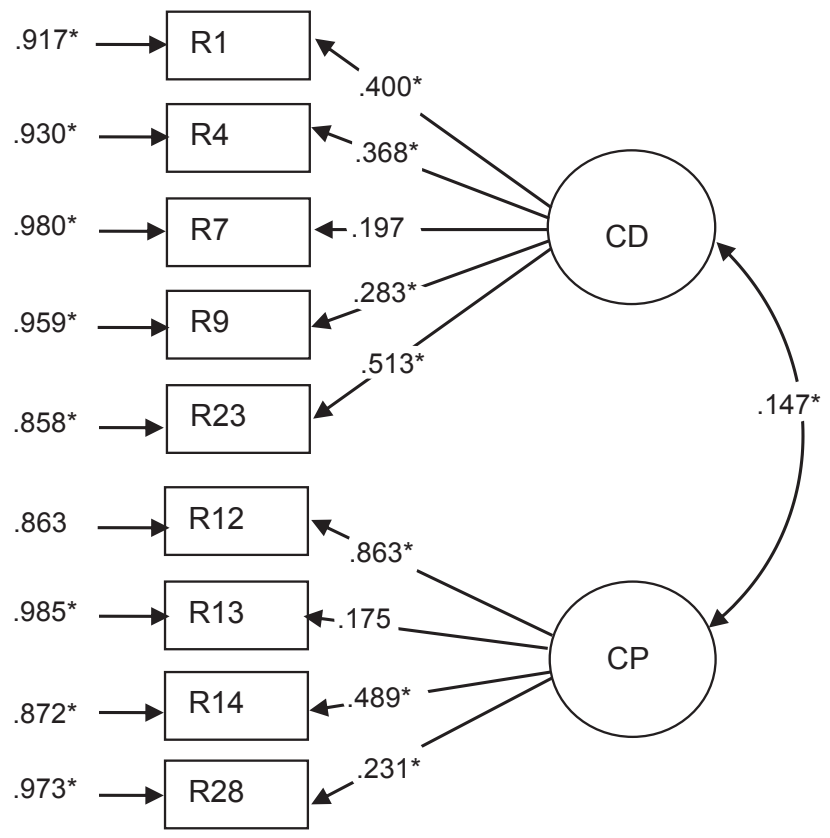

Nota. $\mathrm{CD}=$ Conocimiento declarativo; $\mathrm{CP}=$ Conocimiento procedimental.

Figura 1. Modelo multifactorial de estrategias para el aprendizaje en estudiantes de educación superior por Tipo de Conocimiento. Para cada variable observada $R^{2}=\left(1\right.$ - error de varianza), $\chi^{2}=$ 12 (23 g.l.), $p=.99 ;$ IBBAN =.829; IBBANN=.999; IAC =.999; RMSEA =.000. * $p<0.05 . N=$ 144. 
o variables latentes no fue significativa, como teóricamente era esperado, y se considera un indicador de la validez de constructo divergente entre ambas medidas. La $\chi 2$ fue igual a 13 (19 g.l.) con una probabilidad asociada igual a .81, los indicadores de bondad de ajuste prácticos también fueron adecuados (Bentler, 2006). El coeficiente de contingencia $R H O$ fue igual a .499 .

Al comparar las puntuaciones medias de los estudiantes de ambas licenciaturas a partir de los 26 reactivos, aceptados en el análisis Rasch, no se observaron diferencias significativas ni en tipos de conocimientos ni entre los dos contextos de recuperación de información. Mientras que al contrastar a los estudiantes de la muestra en estudio, a partir de los reactivos que integraron los modelos estructurales, se encontraron diferencias estadísticas a favor de los estudiantes de ingeniería $(M=.462)$ sobre los estudiantes de psicología $(M=.280)$, en cuanto al conocimiento de tipo procedimental: También existen diferencias significativas $(t=$ -2.280, $p=.024$ ) a favor de los estudiantes de ingeniería $(M=.476)$, cuando se les comparó contra los estudiantes de psicología $(M=.394)$ en el Contexto de recuperación de recuerdo o difícil.

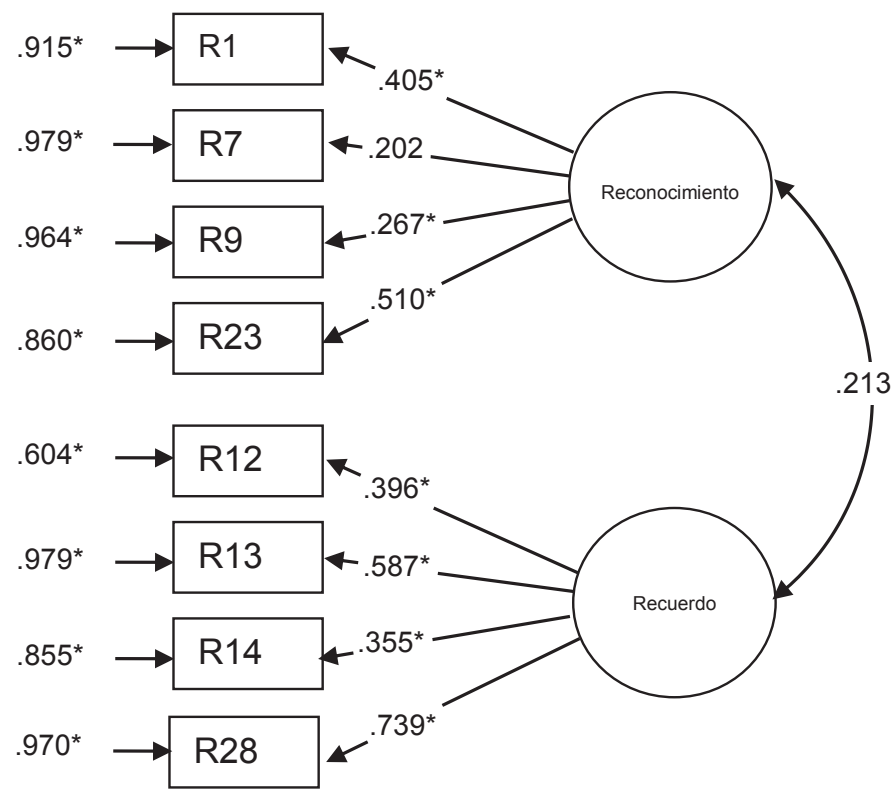

Figura 2. Modelo multifactorial de estrategias para el aprendizaje en dos contextos de recuperación de la información en estudiantes de educación superior. Para cada variable observada $R^{2}=(1$ - error de varianza), $\chi^{2}=23$ (19 g.l. $) p=.20 ; I B B A N=.998 ; I B B A N N=.999 ; I A C=.999 ; R M S E A=.049$ $(0.000, .103) . * p<.05 . N=144$. 


\section{Discusión}

Se logró construir una prueba de ejecución que evalúa los aprendizajes relacionados con el espacio curricular Estrategias para Aprender a Aprender de una universidad pública, a través del Modelo Multidimensional de Evaluación para el Aprendizaje de Castañeda (1998, 2006), mismo que permitirá apoyar la atención de los avances o progresos de los estudiantes, así como detectar dificultades en sus aprendizajes. Además los docentes podrán diseñar o rediseñar las estrategias instruccionales que garanticen una retroalimentación personalizada con el estudiante en cuanto al desarrollo de estrategias de aprendizajes para su mejor desempeño y adaptación al medio académico en la universidad (López-Olivas, 2007) y, posteriormente, en el campo laboral donde se requiera la transferencia de los aprendizajes adquiridos durante su formación universitaria.

Si bien es cierto que la unidimensionalidad implica que un solo rasgo latente se encuentra en la base del conjunto de los reactivos (Hattie, 1985), Linacre y Wright (1989) señalan que, en la práctica, ningún instrumento puede ser perfectamente unidimensional; circunstancia traducible en la práctica a la construcción de instrumentos que en esencia muestren unimensionalidad (Burga et al., 2009). En este sentido, se sugiere atender las dos medidas más precisas de unidimensionalidad de una escala: Los parámetros de ajuste externo e interno para la toma de decisiones sobre la sustitución de reactivos (González, 2008; Wrigth \& Masters, 1982) o bien la presencia de un factor o componente "dominante" que refleje la variable latente medida (Chávez \& Saade, 2009) y realizar análisis de componentes principales de los residuos, luego de ajustar el modelo Rasch unidimensional (Linacre, 2002).

A pesar del alto grado de fiabilidad (.97) arrojado por la prueba KR-20, es importante identificar qué involucra tal consistencia de los reactivos. A partir de los datos arrojados por el análisis de correlación de punto biserial, se demostró baja-nula $(\leq .3)$ correlación de 100\% de los reactivos, lo que no compromete la consistencia de la escala, pero sí la validez del constructo que mide; pese a la demostración de valores de discriminación aceptables $(\geq .80)$, muestran que discriminan en el aspecto particular que miden y no en la globalidad de la escala. Adicionalmente, recordemos que han sido excluidos dos reactivos con nula variabilidad donde la respuesta fue 0 (reactivo 18 y 26).

Es relevante notar que la varianza explicada por los parámetros sugeridos por el modelo: dificultad y habilidad, es apenas de $18 \%$, mientras que la varianza cuya propiedad no explica el modelo es mayor (26\%). Lo anterior tiene muy posiblemente origen además en los aspectos de redacción en condiciones de aplicación.

Al contrastar las puntuaciones medias de los reactivos que miden la recuperación de 
información en el contexto de reconocimiento no hay diferencias estadísticas. Lo cual indica que la prueba construida a partir del MEE es más sensible y contiene menos reactivos que la prueba de 26 reactivos.

Se recomienda seguir en la construcción y prueba de reactivos que midan conocimiento declarativo (identificar) en el contexto de recuerdo o difícil, así como elaborar reactivos que den cuenta de la evaluación de contenidos de tipo procedimental (traducir, inferir y aplicar) en el contexto de reconocimiento, con el propósito de fortalecer y mejorar la prueba EPA lograda hasta este momento; es de primordial importancia la definición clara y explícita del objeto de medición (López-Olivas, 2007), para que oriente de manera óptima los procesos de su medición.

Finalmente, se concluye que la prueba de EPA construida con base en el modelo multidimensional de evaluación de Castañeda (1998, 2004), permite identificar los efectos diferenciales de las demandas asociadas con los diversos niveles de complejidad subyacentes tras la ejecución en dos contextos de prueba. Como afirma Castañeda (2004, 2006), es una herramienta evaluativa sensible a la detección de fallas y apoya la prescripción oportuna de técnicas remediales específicas e individualizadas. De esta forma, se desarrollará la capacidad para determinar qué, cómo y en qué tipo de tarea un estudiante dado falló y se dará la instrucción remedial necesaria o se rediseñarán las condiciones del aprendizaje (Castañeda, 2006) en el espacio curricular EAA del nivel de licenciatura. Asimismo, se estará en mejores condiciones para facilitar y garantizar la transferencia de aprendizajes a otros espacios curriculares, así como al contexto laboral.

\section{Referencias}

Aguilar, J., Pacheco, J., Andrade, J., Vargas, A., Gutiérrez, P., \& Zetina, G. (1992). Estudio sobre la validez concurrente del inventario de factores de carrera en estudiantes de licenciatura. Monografía. México: UNAM.

Araoz, R. M., Guerrero, P., Galindo, M., Villaseñor, R., \& De la Vara, A. (2010). Estrategias para aprender a aprender. México: Pearson.

Bentler, P. M. (2006). EQS structural equations program manual. Los Ángeles, CA: BMPD Statistical Software. Inc.

Burga, A., Llanos, F., Pérez-León, H., Simon, J., Boccio, K., Miranda, L., Arias, M., Pacheco, T., \& Asmad, U. (2009). Evaluación censal de estudiantes (ECE). Segundo grado de primaria y cuarto grado de primaria de IE EIB. Marco de trabajo. Ministerio de Educación del Perú. Recuperado de http://www2.minedu.gob.pe/umc/ece/ Marco_de_Trabajo_ECE.pdf

Cadavid, N., Delgado, A., \& Prieto, G. (2007). Construcción de una escala de depresión con la escala de Rasch. Psicotema, 19(3), 
515-521. España: Universidad de Oviedo. Recuperado de http://www.redalyc.org/ articulo.oa? $\mathrm{id}=72719323$

Castañeda, F. S. (1993). Procesos cognitivos y educación médica. México: UNAM.

Castañeda, F. S. (1998). Evaluación de resultados de aprendizaje en escenarios educativos. Revista Sonorense de Psicología, 12, 57- 67.

Castañeda, F. S. (2004). Educación, aprendizaje y cognición. En S. Castañeda (Ed.), Educación, aprendizaje y cognición. Teoría en la práctica (pp. 49-74). México: Manual Moderno.

Castañeda, F. S. (2006). Evaluación del aprendizaje en el nivel universitario. Elaboración de exámenes y reactivos objetivos. México: UNAM-Conacyt. ISBN 970-32-3960-9.

Chávez, A., \& Saade, H. (2009). Procedimientos básicos para el análisis de reactivos. Cuaderno Técnico 8. CENEVAL. México. Recuperado de http:/www.ceneval.edu.mx/ ceneval-web/content.do?page $=1689$

De Jong, F., \& Simons, P. (1990). Cognitive and metacognitive processes of self-regulated learning. En J. Pieters, P. Simons \& L. Leeuw (Eds.), Research on computer-based instruction (pp. 81-100). Amsterdan: Swets \& Zeitlinger.

González, D., Valenzuela, M. E., \& Maytorena, N. M. (1999). La estructura factorial de la inseguridad vocacional: Un estudio confirmatorio. Enseñanza e investigación en psicología, 3, 163-183.
González, M. M. J. (2008). El análisis de reactivos con el modelo rasch manual técnico. Instituto Nacional para la Evaluación de la Educación-Universidad de Sonora. Recuperado de http:/www.winsteps.com/a/ recursos-offline.pdf

González, L. D., Castañeda, F. S., \& Maytorena, N. M. (2009). Estrategias referidas al aprendizaje, la instrucción y la evaluación. México: Pearson.

Hambleton, R. K., \& Swaminathan (1985). Item response theory. Principles and applications. Boston, MA: Kluwer Nijhoff Publishing.

Hattie, J. (1985). Methodology review: Assessing unidimensionality of tests and items. Applied Psychological Measurement, 9(2), 139-164.

Linacre, J. M. (1991). Structured rating scales. Sixth international objective measurement workshop. Chicago. Recuperado de http:// www.winsteps.com/a/Linacre-structuredrating-scales.pdf

Linacre, J. M. (2002). Optimizing rating scale category effectiveness. Journal of Applied Measurement, 3(1), 85-106. MESA Psychometric Laboratory. University of Chicago. Recuperado de http://www. winsteps.com/a/linacre-optimizing-category. pdf

Linacre, J. M. (2006). A user's guide to winsteps ministeps: Rasch-model computer programs. Chicago, USA. Recuperado de http://www. winsteps.com 
Linacre, J., \& Wright, B. (1989). Length of a logit. Rasch measurement transactions, 3(2), 54-55. Recuperado de http://www.rasch.org/ $\mathrm{rmt} / \mathrm{rmt} 32 \mathrm{~b} . \mathrm{htm}$

López-Olivas, M. (2007). El modelo de alumno deseable para la institución ¿es el que refleja el modelo de examen de admisión? En D. González \& M. Maytorena (Eds.), Estudios Empiricos en Educación Superior (pp. 1935). México: Universidad de Sonora. ISBN: 970-689-351-2.

Martínez-Guerrero, J., \& Sánchez-Sosa, J. J. (1993). Estrategias de aprendizaje: análisis predictivo de hábitos de estudio en el desempeño académico de alumnos de bachillerato. Revista Mexicana de Psicología, 10(1), 63-73.

Prieto, G., \& Delgado, A. (2003). Análisis de un test mediante el modelo de rasch. Psicothema. 15(1), 94-100. Recuperado de http://www. psicothema.com/psicothema.asp?id=1029

Revuelta, J., \& Ponsoda, V. (1998). Un test adaptativo informatizado de análisis lógico basado en la generación automática de ítems. Psicothema, 10(3), 709-716. Recuperado de http://www.psicothema.com/psicothema. asp?id=199

Tirado, F., González M. M. J., \& Backhoff, E. (2009). Procesos de control de calidad del examen de habilidades y conocimientos básicos (EXHCOBA). Memorias del $X$ Congreso Nacional de Investigación Educativa. Veracruz, Ver.
Universidad de Sonora (2004). Eje de Formación Común. Programa de estrategias para el aprendizaje. Recuperado de http://www. uson.mx/institucional/marconormativo/ reglamentosacademicos/eje_formacion_ comun.htm

Valle, A., González, R., Cuevas, L., \& Rodríguez, S. (2003). Las estrategias de aprendizaje: Características básicas y su relevancia en el contexto escolar. España. Universidad de la Coruña. Departamento de la Psicología Evolutiva y de la Educación. Recuperado de htpp:/www.educadormarista. com/Descognitivo/ESTRAPE4.HTM

Wright, B. D., \& Masters, G. N. (1982). Rating scale analysis. Chicago: MESA Press.

Wright, B. D., \& Stone, M. H. (1979). Best test design. Chicago: MESA Press. ISBN 0-941938-00-X. 
ANEXO: Estrategias para el Aprendizaje*

Instrucciones: Lee todos los reactivos que conforman la prueba y responde lo que te solicita cada uno de ellos, marca con una cruz la opción que consideres correcta. NO es necesario que anotes tu nombre ni algún dato personal.

Ejemplo:

1. ¿Cuál es la capital de Sonora?
a) Ures
b) Álamos
c) Hermosillo
d) Cd. Obregón

1. Se refiere a los rasgos cognitivos, afectivos y fisiológicos que sirven para conocer la forma en que los estudiantes responden a sus ambientes de aprendizaje:
a) Aprender a aprender
b) Estrategias de aprendizaje
c) Estilos de aprendizaje
d) Cognición

Justificación: c) se refiere a rasgos, no a actividades ni a procesos.

2. ¿Cuál es el proceso que señala el papel activo que el estudiante debe desempeñar en su aprendizaje?

a) Cognición

b) Estilos de aprendizaje

c) Aprender a aprender

d) Estrategias de aprendizaje

Justificación: c) Procedimiento personal más adecuado para la adquisición y generación de conocimiento.

3. Proceso que permite organizar el conocimiento, planear las actividades cognoscitivas, controlar el proceso intelectual y evaluar los resultados:
a) Metacognición
b) Cognición
c) Planeación estratégica
d) Estrategias de aprendizaje. 
Justificación: a) es la correcta, ya que este proceso permite desarrollar potencial de aprendizaje y favorece el aprender a aprender mediante la planeación, adquisición, desarrollo y uso adecuado de estrategias.

4. La afirmación "en el aula el estudiante aprende partiendo de sus experiencias y conocimientos previos", es una característica del aprendizaje llamada:

a) Acumulativo

b) Constructivo

c) Situado

d) Colaborativo.

Justificación: a) es la respuesta correcta, ya que dentro de la teoría del constructivismo se afirma que el conocimiento nuevo se construye sobre la base del conocimiento previo.

5. ¿Cuál es el único estilo de aprendizaje relacionado con seleccionar y representar la información?

a) Visual

b) Pragmático

c) Reflexivo

d) Teórico

Justificación: a) es el estilo de aprendizaje que caracteriza a una persona que establece relaciones entre ideas y conceptos, piensa en imágenes y gusta de las descripciones con la información a aprender.

6. ¿Cuál es el estilo de aprendizaje que define a una persona que analiza datos antes de llegar a conclusiones?
a) Reflexiva
b) Visual
c) Activa
d) Pragmática.

Justificación: a) es la correcta, ya que es el estilo de aprendizaje que caracteriza a una persona que considera las experiencias y las observa desde diferentes perspectivas, al procesar la información para aprenderla.

7. ¿Cuáles son los factores que se refieren a organizar nuestras ideas, nuestro tiempo y los factores físicos que nos rodean al estudiar? 

a) Ambientales y de organización
b) Afectivo-social
c) Educativos
d) Cognoscitivos

Justificación: a) es la correcta, ya que en estos factores quedan incluidos aquellos elementos del medio ambiente que inciden positivamente o negativamente en la calidad del estudio que se lleva a cabo.

8. ¿Qué es lo característico en el empleo de una técnica de lectura?
a) Subrayar
b) Anotar dudas
c) Memorizar
d) Aprender algo

Justificación: d) es la correcta, ya que las opciones a) y b) son técnicas de lectura, no una característica de la técnica de lectura; la opción c) no corresponde con el fin de leer.

9. ¿Cuál de las siguientes características corresponde al proceso de esquematización?
a) Dialogar con el autor
b) Destacar lo más importante del texto
c) Ordenar las ideas aprendidas
d) Apoyar nuestra memoria

Justificación: c) es la correcta, ya que las opciones a) y d) son funciones de las notas al margen; la opción b) hace referencia al subrayado.

10. A qué tipo de esquematización le corresponde la siguiente definición "construir, registrar, organizar y asociar ideas como las procesa el cerebro humano"
a) Esquema analítico
b) Mapa conceptual
c) Mapa mental
d) Cuadro sinóptico

Justificación: Todas las opciones son ejemplo de esquematización, pero el reactivo corresponde a la definición específica de mapa mental. 
11. ¿De los siguientes procesos cuáles corresponden a las operaciones de pensamiento?

a) Observación, comparación y relación y clasificación

b) Interacción entre lenguaje y pensamiento

c) Hojear, examinar, subrayar

d) Comprender, interpretar, reflexionar

Justificación: a) es la correcta, ya que la opción b) corresponde al segundo enfoque teórico del proceso de lectura; la opción c) son funciones de la lectura de selección y la opción d) hace referencia a la lectura analítica.

12. Elabora un mapa conceptual a partir del siguiente texto:

El concepto de eficacia biológica se refiere al grado de éxito con que un individuo consigue transmitir sus genes a la siguiente generación. La forma tradicional de medir la eficacia biológica ha sido mediante el número de descendientes, puesto que es la forma más obvia y directa de transmitir los propios genes. Más recientemente, sin embargo, la genética ha ampliado el antiguo concepto de eficacia biológica. Según la posición genetista, expresado en términos metafóricos, es cada gen el que busca replicarse e incrementar su frecuencia en la siguiente generación. Dado que cualquier individuo está genéticamente relacionado con muchas personas además de sus propios hijos, un gen puede incrementar su frecuencia en la población modificando el comportamiento del individuo hacia los propios parientes en su totalidad. Si un gen induce en una persona un comportamiento que potencie la eficacia biológica de sus parientes, el gen (que es muy probable que esté presente en dichos parientes) incrementará su número en la siguiente generación.

Los teóricos evolucionistas distinguen los conceptos de eficacia biológica individual y eficacia biológica inclusiva. La primera hace referencia al nivel de éxito de mis genes en la lucha por la existencia medida a través de mi propia descendencia; cuanta mayor es la descendencia, mayor es el nivel de eficacia biológica individual. Mientras que la eficacia biológica inclusiva agrega a esto una proporción de mi eficacia biológica medida a través de mis efectos sobre mis parientes. En la medida en que mis genes me lleven a contribuir a la supervivencia de mis parientes y aumenten su descendencia, aumentaré mi eficacia biológica inclusiva. La eficacia biológica inclusiva comprende la eficacia biológica individual y es la medida de la eficacia biológica total de mis genes en la lucha por la supervivencia.

Otra forma de definir la eficacia biológica inclusiva puede distinguirse entre directa e indirecta eficacia biológica. Un individuo recibe eficacia biológica directamente de sus progenitores y la 
aporta directamente de otros, ya sea a través de comportamientos seleccionados por el parentesco a través de la reciprocidad, y los aporta a otros, sean parientes (selección de parentesco) o no parientes (reciprocidad). Las expresiones de eficacia biológica directa e indirecta nos ayuda a ver que la eficacia biológica inclusiva abarca efectos que pueden no estar basados en el parentesco genético, sino que comprende además la ayuda o el daño -prestada o infligido- a no parientes. Así pues, la eficacia biológica inclusiva comprende la eficacia biológica que heredamos de nuestros parientes (eficacia biológica individual y eficacia biológica seleccionada por el parentesco) y la eficacia biológica que acumulamos de interacciones con no parientes.

El concepto de eficacia biológica inclusiva lleva a la idea de selección por parentesco. Un gen cuya estrategia sea sólo potenciar la eficacia biológica individual, quizá haciendo que un individuo quiera y tenga mucha descendencia, está sujeto a selección individual: el que tenga éxito o el que fracase depende de la vida y la muerte de la máquina de supervivencia que lo alberga. Un gen cuya estrategia sea la de inducirme a ayudar a mis parientes (y de ese modo, ayudarse a sí mismo a replicarse a través de ellos) está sometido a selección por parentesco: el que tenga éxito o el que fracase depende menos de mi propia vida o de mi muerte que de la medida mediante la cual mi sacrificio ayude a mi parentela. La lógica de la selección por parentesco es fundamental para el análisis del altruismo a través de la psicología evolucionista.

[Hardy, T., \& Jackson, R. (1998). Aprendizaje y cognición. Madrid: Prentice Hall]

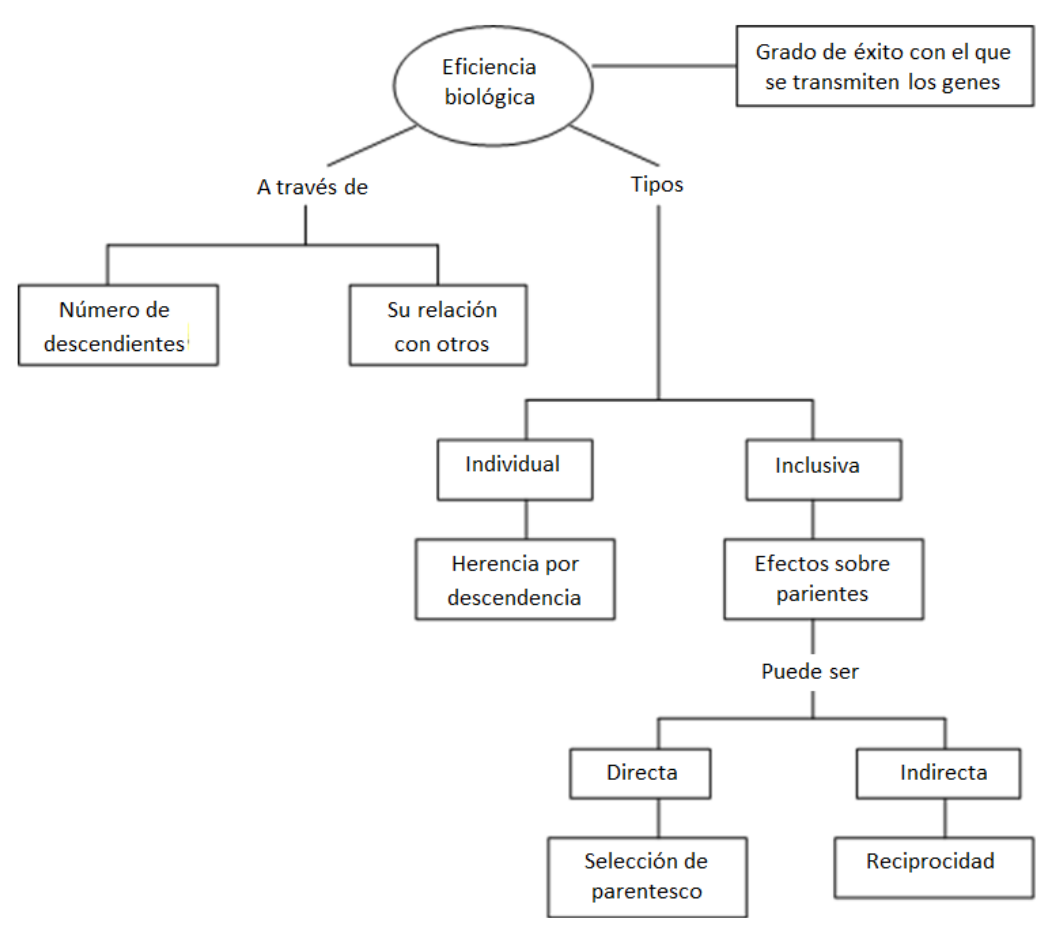


13. ¿Cuál sería un título adecuado para el texto anterior? Eficacia Biológica

Justificación: Eficacia biológica es la idea principal del texto

14. A partir del esquema que elaboraste reconstruye el texto con base en los lineamientos para la redacción de un texto.

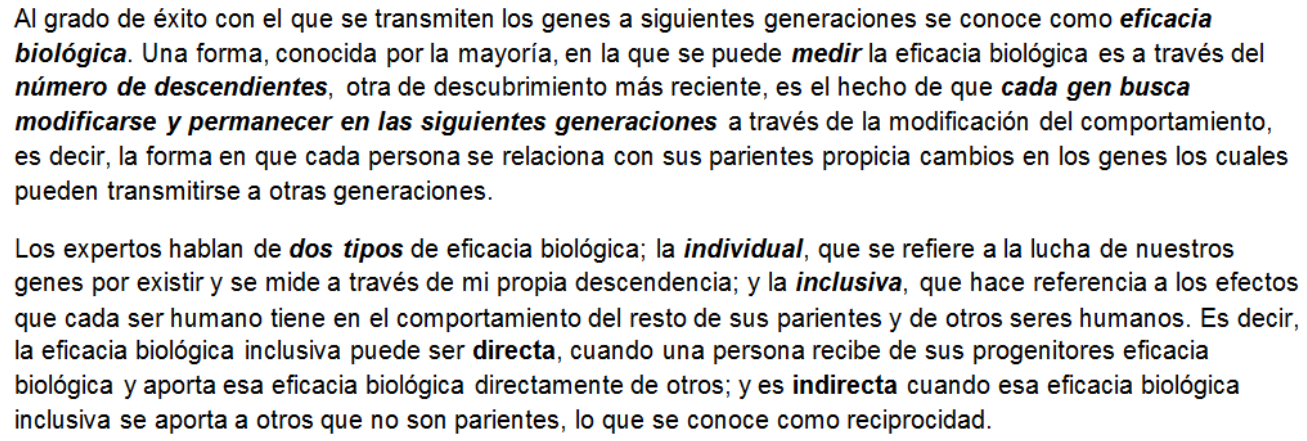

15. Elabora una inferencia a partir del texto: "Al establecer una nueva sociedad en América, los españoles enfrentaron una serie de dilemas. El más importante era qué hacer con los habitantes originarios, a quienes llamaron indios. El dilema discurría acerca de la naturaleza de los indios: si eran humanos o no".

\section{Justificación:}

$\mathrm{R}_{1}$ : Luego de la conquista, los indígenas no eran considerados como seres humanos por los españoles.

$\mathrm{R}_{2}$ : El no considerar a los indios como seres humanos, provocó conflictos entre las autoridades españolas cuando intentaron estructurar la nueva sociedad.

$\mathrm{R}_{3}$ : Los españoles no sabían qué hacer con los habitantes, si eran humanos o no.

16. ¿Cuál sería el título más adecuado para el texto de la pregunta 15 ?

\section{Justificación:}

$\mathrm{R}_{1}$ : Los dilemas de los españoles después de la conquista.

$\mathrm{R}_{2}$ : La naturaleza de los indios después de la conquista.

$\mathrm{R}_{3}$ : El dilema más importante de los españoles después de la conquista.

$\mathrm{R}_{4}$ : La visión que los españoles tenían de los indios. 
17. "La reforma educativa que se está proponiendo para este país será un fracaso. Esta reforma corresponde en sus puntos esenciales a la reforma francesa, cuyos resultados fueron desastrosos. La reforma francesa tampoco se diferencia de las que se realizaron en Bélgica y Dinamarca, y éstas también fracasaron. Si la reforma educativa que se implantó en estos tres países fracasó, también fracasará en este país". ¿Qué tipo de argumentación se utiliza en el texto anterior?:

a) Analógico inductivo

b) Analógico deductivo

c) Argumento deductivo

d) Argumento inductivo

Justificación: a) es la respuesta correcta porque la relación entre el supuesto, las evidencias y la conclusión es probable, es decir, es un argumento inductivo y; al comparar la aplicación de la reforma educativa en otros países con la implantación de la misma en este país, es por ello también un argumento analógico.

18. En el texto: "Quisiera defender la tesis de que la tarea más urgente de los estudios culturales es plantear los lineamientos para una crítica de la economía política de la cultura, (a nuestra) disposición se encuentra toda una tradición de pensamiento crítico elaborada durante el siglo pasado, (dentro de) la cual la obra de Althusser contribuyó de manera significativa. Obviamente, esta tradición deberá ser repensada y reelaborada según las nuevas necesidades de la sociedad contemporánea". ¿Qué argumento se puede refutar?:

Justificación: El autor citado como autoridad (Althusser) ya no es vigente.

19. "El amor de la madre significa dicha, paz, no hace falta conseguirlo ni merecerlo. La relación con el padre es enteramente distinta”. ¿Por qué el argumento expuesto es una falacia?:

\section{Justificación:}

$\mathrm{R}_{1}$ : El autor supone una figura ideológica de la madre y la contrasta con una figura ideológica del padre.

$\mathrm{R}_{2}$ : El autor se basa en una postura tradicional que asigna roles específicos tanto al padre como a la madre. Es decir, patrones ya establecidos.

$\mathrm{R}_{3}$ : Se basa en roles establecidos. 
20. Texto mediante el cual se expone, analiza, reflexiona, argumenta y comenta una interpretación personal sobre un tema determinado:
a) Ensayo
b) Hipertexto
c) Apéndice
d) Resumen

Justificación: a) es la opción correcta, ya que abarca las características antes mencionadas, el término Hipertexto hace hincapié en la creación de un texto a partir de otros, no en la rigurosidad y presentación de la opinión original del autor, la autoría... el resumen es eso, un "compacto" o descripción en pocas palabras de lo esencial de un escrito, y el apéndice literalmente es sólo una "cosa adjunta o añadida a otra, de la cual es como parte accesoria o dependiente.

21. Marca la opción que enlista la serie de características que exige el ensayo:

a) El ensayo exige una organización adecuada de las ideas planteadas, así como un léxico directo y preciso, además de profundidad en la reflexión y propuesta el autor, así como de citas que respalden a la misma.

b) El ensayo exige una adecuada organización de las diversas posturas, el léxico puede ser superficial e indirecto, ya que no es relevante para la hipótesis del autor.

c) El ensayo no exige una hipótesis propia del autor, si bien si es necesario un léxico directo y profundo, la organización de las ideas es el eje central de la propuesta.

d) Si bien, exige una buena organización de las ideas planteadas, no requiere de profundidad, ni de las citas que respalden lo referido en el trabajo.

Justificación: a) el ensayo requiere rigor del pensamiento y de la organización adecuada de las partes, utilizar un léxico directo y preciso con preguntas que generen expectativas y con citas que respalden las reflexiones y argumentos del autor. Así, el autor debe investigar diversas fuentes acerca del tema, esto le dará solidez a la argumentación de la hipótesis propuesta.

22. ¿Por qué se afirma que el ensayo "expone el proceso de pensar"?:

a) El autor se basa en otro autor y a partir de éste le da sentido a una nueva problemática, repite los argumentos de ese autor de una manera exhaustiva, ordenada, clara y sencilla.

b) El autor recopila una serie de argumentos alrededor de un problema y los organiza por orden de importancia, es claro y sencillo. 
c) El autor propone su punto de vista con respecto a un problema, es nuevo, y por lo tanto diferente a los otros, es original y claro.

d) El autor propone un punto de vista con respecto a un problema, el autor se basa en otro autor y, así le da sentido a una nueva problemática.

Justificación: c) por que a partir de la revisión exhaustiva, el autor se aventura a proponer una nueva visión de la problemática, rebasando la mera exposición de "hechos" relativos al tema, es original, este detalle central no aparece en a), ni en b), c) es una variante de a) que no incluye la originalidad o lo propositivo.

23. Cuáles son los elementos del ensayo como género discursivo:

a) Introducción, problemática, antecedentes, la postura del autor y la conclusión.

b) Introducción, la postura del autor y la conclusión.

c) Introducción, la lectura en grupo, la problemática y la conclusión del autor.

d) Introducción, la lectura en grupo, la postura del autor y la conclusión.

Justificación: a) es la respuesta correcta, las demás opciones tienen omisiones y/o errores.

24. Marca la opción que enlista en orden los pasos a cubrir para realizar las redes semánticas:

a) 1. Elaborar un mapa conceptual a partir de la lectura del texto; 2. Elaborar redes semánticas a partir de los ejes verticales o importantes de la lectura, 3. Redactar los argumentos usando los conectores apropiados y 4. Elaborar la introducción y la conclusión cuidando no cometer errores gramaticales.

b) 1. Elaborar redes semánticas a partir de los ejes verticales o importantes de la lectura, 2. Redactar los argumentos usando los conectores apropiados, 3. Elaborar un mapa conceptual a partir de la lectura del texto y 4. Elaborar la introducción y la conclusión cuidando no cometer errores gramaticales.

c) 1. Elaborar un mapa conceptual a partir de la lectura del texto, 2. Elaborar la introducción y la conclusión cuidando no cometer errores gramaticales, 3. Redactar los argumentos usando los conectores apropiados y 4. Elaborar redes semánticas a partir de los ejes verticales o importantes de la lectura.

d) 1. Elaborar redes semánticas a partir de los ejes verticales o importantes de la lectura, 2. Elaborar un mapa conceptual a partir de la lectura del texto, 3. Redactar los argumentos usando los conectores apropiados y 4. Elaborar la introducción y la conclusión cuidando no cometer errores gramaticales.

Justificación: a) es el orden correcto de los pasos a seguir para elaborar las redes semánticas. 
25. ¿Cuál es la definición del término hipertexto en la lectura como estrategia cognoscitiva y metacognoscitiva?

a) El autor escribe un texto nuevo con información de otro autor, agotando la complejidad del problema a estudiar.

b) El autor resume con claridad los diversos puntos de vista sobre un tema y los presenta en un esquema de clasificación, ayudándose de mapas conceptuales

c) El autor escribe un texto nuevo con información parcial de un solo autor, esto es, sin agotar la complejidad del problema a estudiar.

d) El autor escribe un texto a partir de otros, por lo tanto, se convierte en un escritor, ya que al analizar profundamente varios puntos de vista, genera una nueva opinión.

Justificación: d) el hipertexto se define como la creación de un texto nuevo a partir de otros, así, el Hipertexto marca los diferentes discursos utilizados por un autor (o varios) para construir otro. El lector se convierte en escritor, ya que necesita comprender, interpretar y conocer varios textos de diversos autores.

26. ¿Cuáles son los tipos de hipertextos que un estudiante puede utilizar como estrategia cognoscitiva y metacognoscitiva?

Hipertexto gráfico e hipertexto escrito.

Justificación: Son las opciones que los autores nos presentan dentro del curso de actividades a desarrollar.

27. Marca la opción que enlista en orden los pasos para realizar un hipertexto:

a) Lectura en grupos del material e identificación de ideas principales, revisar individualmente y comentar lo leído, repetir la lectura y las discusiones y decidir el nombre de la problemática elegida. b) Lectura en grupos del material e identificación de ideas principales, revisar individualmente y comentar lo leído y decidir el nombre de la problemática elegida.

c) Lectura en grupos del material, revisar individualmente y comentar lo leído, repetir la lectura y las discusiones y decidir el nombre de la problemática elegida.

d) Lectura en grupos del material, decidir el nombre de la problemática elegida, elaborar un mapa conceptual y escribir claramente.

Justificación: a) es la correcta porque presenta en orden los pasos para realizar un hipertexto. 
28. Elabora un breve ensayo partiendo de la estrategia del HIPERTEXTO, para esto, te presentamos 2 lecturas, la primera aparece en el reactivo 12, la segunda se te presenta a continuación:

Los biólogos utilizan la expresión eficacia biológica (o aptitud) para describir la capacidad de un genotipo determinado para dejar descendientes en la siguiente generación en relación con la capacidad de otros genotipos de hacerlo. Por lo tanto, si los escarabajos marrones sistemáticamente producen más descendientes que los escarabajos verdes debido a su color, podría decirse que los escarabajos marrones tienen una eficacia biológica más alta.

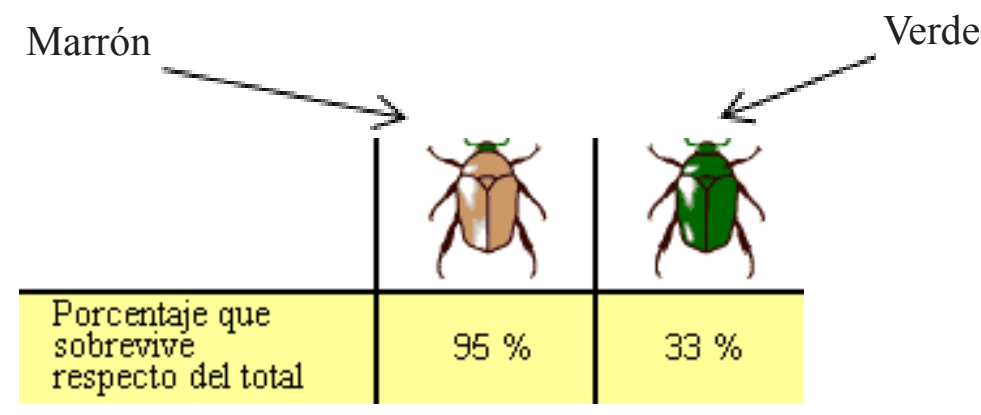

Los escarabajos marrones tienen una eficacia biológica superior con relación a los escarabajos vierdes.

Por supuesto, la eficacia biológica es relativa. La aptitud de un genotipo depende del ambiente en que vive y el genotipo más apto durante una glaciación, por ejemplo, probablemente no sea el genotipo más apto una vez que la glaciación ha terminado.

La eficacia biológica es un concepto que resulta muy útil porque reúne en una sola idea todo lo que importa en la selección natural (la supervivencia, encontrar pareja, la reproducción). El individuo más apto no es necesariamente el más fuerte, el más rápido ni el más grande. La eficacia biológica de un genotipo incluye su capacidad de sobrevivir, encontrar una pareja, producir descendientes y, en última instancia, dejar sus genes en la siguiente generación.
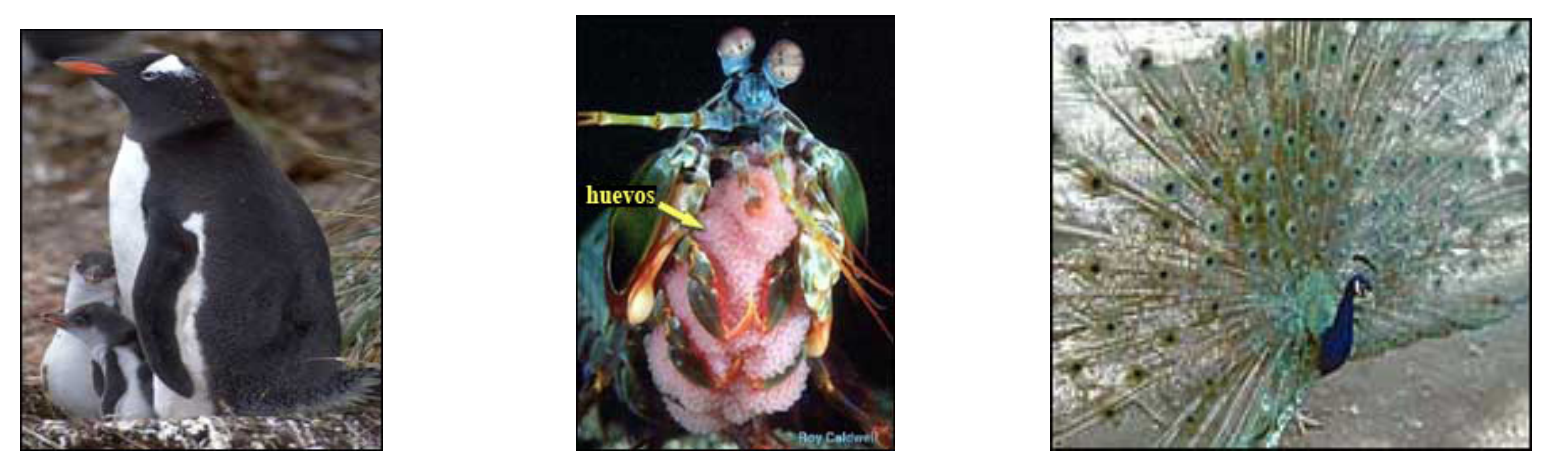
Cuidar de los descendientes (arriba a la izquierda); producir miles de crías, muchas de las cuales no sobrevivirán (arriba al centro); o lucir plumas llamativas que atraigan a las hembras (arriba a la derecha) son un problema para la salud y la supervivencia del progenitor. Sin embargo, estas estrategias aumentan la eficacia biológica porque ayudan a que los progenitores consigan tener un mayor número de descendientes en la siguiente generación.

\section{Justificación:}

1) Contenidos importantes Lectura 1 (Eficacia Biológica, EB)

- Definición de E B,

- Cómo identificarla (a través de la reproducción del sujeto, o a través de ejercer influencia sobre otros)

- Tipos: Individual e inclusiva (directa e indirecta)

- La idea de selección por parentesco

2) Contenidos importantes de lectura 2 (qué hay de la EB)

- Definición, la relatividad de ésta a partir del medio ambiente

- La idea de un término integrador en la E B

- Las diversas estrategias para la de selección

(*) Prueba elaborada por Daniel González-Lomelí, María de los Ángeles Maytorena-Noriega y César Varela-Romero, académicos del Departamento de Psicología y Ciencias de la Comunicación de la Universidad de Sonora. Proyecto SEP-CONACYT; CB-2011-01; Clave: 000000000167235; Modalidad: G3. 